\title{
Truth About: Coronavirus (COVID19)
}

Athar $\mathbf{M}^{*}$

Assistant Professor, Department of Forensic Science, Institute of Excellence in Higher Education, India

Perspective Article

Volume 5 Special Issue 1

Received Date: April 22, 2020

Published Date: June 09, 2020

*Corresponding author: Mohammad Athar, Assistant Professor, Department of Forensic Science, Institute of Excellence in Higher Education, Bhopal, India, Tel: 9826047806; Email: athar.fsl48@gmail.com

\section{What is a Virus?}

Viruses are tiny and relatively simple. They exist as scraps of genetic code wrapped in a protein and lipid (fat) overcoat, measuring about one thousandth of the diameter of a human hair (15-300) nanometers across and much smaller than bacteria). It is debatable whether viruses are living, since they hijack the cells in our bodies to create more viruses by turning the cells into virus factories. This is why viral infections-such as the common cold - are so tricky to treat: they're hard to combat without also damaging the cells the virus has infected.

\section{What is a Coronavirus?}

This pandemic of coronavirus disease (COVID-19) is caused by severe acute respiratory syndrome coronavirus i2 (SARS-CoV-2).

The name coronavirus is derived from the Latin corona, meaning "crown" which irefers to the characteristic halo of club-shaped spikes of protein that the virus uses to invade human cells. The coronavirus measures around 125 nanometers across and consists of 30 genes (by comparison, humans have 20,000). The virus can be seen under an electron microscope.

\section{The Pandemic: What is the Current State of the Pandemic?}

Scientist's estimate that the virus is capable of causing 40 million deaths worldwide if left unchecked. Over the centuries there have been a number of pandemics across the globe, such as smallpox (caused by a virus) and the bubonic plague (caused by bacteria).

\section{What are Government Measures trying to achieve?}

UK government measures are aimed at preventing the
NHS from being overwhelmed by a sharp spike in COVID-19 that would have catastrophic knock-on effects, notably on other seriously-ill patients. The aim is to suppress the epidemic, spreading the burden out for longer so the peak demand is much lower. However, timing draconian measures to blunt the epidemic is tricky because they have significant health, social and economic downsides - introduce them too early and you get the downside without much upside.

New cases of COVID-19 have slowed dramatically in China, but some fear that once the country relaxes its control measures, the virus could start circulating again. The idea that the pandemic can be contained and 'will go away...does not strike me as plausible,' says Prof Chris Whitty, Chief Medical Officer for England (CMO).

\section{Why do Scientists Seem to be Arguing about how to Curb the Pandemic?}

That's the way science works - theory and ideas are endlessly challenged by data, new ideas and the results of experiments. When there is a conflict between evidence and theory, a new, better theory results.

When it comes to the pandemic, there have been arguments about the epidemiology - the branch of medicine which assesses the incidence, distribution and control of a disease - whether by computer models to predict the course of the pandemic or the efforts of 'shoe-leather epidemiologists' who do painstaking investigations to trace infected people. Because this is a novel disease our 'learning is evolving all the time,'says Sir Patrick Vallance, Chief Scientific Adviser (CSA). The Chief Medical.

\section{How long will this Pandemic Last?}

Computers can be used to make predictions, though they are entirely dependent on the current state of knowledge (as they warn in computer science, 'garbage in, garbage out'). The 


\section{Open Access Journal of Pulmonary \& Respiratory Sciences}

influental coronavirus epdemic modeling report (summary) by epidemiologists Neil Ferguson, Azra Ghani, Christl Donnelly et al at Imperial College's Medical Research Council Centre for Global Infectious Disease Analysis suggests (see figures 3 or 4) the epidemic will continue for several months at least as measures are taken to suppress the outbreak.

However, the Imperial Team Emphasizes: There are very large uncertainties around the transmission of this virus, the likely effectiveness of different policies and the extent to which the population spontaneously adopts risk-reducing behaviors. This means it is difficult to be definitive about the likely initial duration of measures which will be required, except that it will be several months. You can get a sense of the power of early intervention by using this epidemic calculator.

\section{Will Covid-19 Return?}

Yes. Another reason for the uncertainty about how the pandemic will develop are seasonal effects, which we don't understand and can be very important. However, high temperature and humidity cut the transmission of COVID-19, suggesting that the arrival of summer and rainy season in the northern hemisphere could damp down the pandemic. Equally, people congregate more in cold weather so it remains unclear if seasonal effects are due to the virus or changes in the way that we behave.

\section{What does Success in Curbing the Pandemic Look Like?}

There are 8000 or so deaths in an average UK influenza season, according to Sir Patrick Vallance. If the coronavirus death rate is kept to a comparable level it will be a 'remarkable achievement' given there is no established vaccine against coronavirus. (Note that Public Health England says the annual average is more like 17,000).

\section{What do the Epidemiologists most want to know?}

What proportion of people catch the virus without symptoms is not known and this knowledge could be transformational, says Prof Whitty. Researchers are racing to develop reliable antibody tests, also called serological tests, which are able to reveal whether someone was infected even after their immune system has cleared the virus that causes COVID-19. The UK government is looking at lab tests and home tests, says Sir Patrick Vallance, which will show who has had the disease without showing symptoms, who won't catch it again and so on. Experts doubt anecdotal reports of people who have had a second COVID-19 infection. Another technology, called PCR, can be used to detect the virus itself.

\section{How else can we Curb the Pandemic without a Drug or Vaccine?}

Coronavirus cases have dropped sharply in South Korea. The secret of its success is the most expansive and well-organized testing program in the world, combined with extensive efforts to isolate infected people and trace and quarantine their contacts. The journal Nature has bemoaned how WHO advice is not being followed, which is to aggressively test, track and isolate as many cases of COVID-19 as possible. However, this strategy is the most powerful - and practical - nearer the start and end of the epidemic, says Prof Whitty.

\section{How do we track the Pandemic?}

The genetic code is the recipe for building a virus and it subtly changes as the virus mutates. By reading the genetic code, using sequencing technology pioneered in Britain by double Nobel Prize-winner Fred Sanger, it is possible to see when an outbreak is local or has been imported from elsewhere.

Today, the UK announced a $£ 20$ million investment in the COVID-19 Genomics UK Consortium to deliver largescale, rapid sequencing and share intelligence with hospitals, regional NHS centres and the Government. Scientists are already crowd-sourcing genomic information on SARS-CoV-2 strains from around the world to track viral evolution and have produced the family tree for the virus.

\section{Coronavirus Infection: How does a Coronavirus infect Human Cells?}

One of the protein spikes on the outside of the virus attaches to a human cell, latching on to a human protein known as angiotensin converting enzyme 2, or ACE2, that is found on cells of the lung, intestine, kidney, and blood vessels.

Scientists have worked out the atomic structure of this spike in various ways which can help develop countermeasures such as vaccines and treatments - for example, there is a citizen science effort to find drugs to bind to the spike. This mechanism could help explain features of the disease: because the manufacture of ACE2 is substantially greater in people with type 1 or type 2 diabetes, it could be that diabetes and hypertension treatment with ACE2stimulating drugs increases the risk of developing severe COVID-19.

After the virus docks with human cells, the fatty overcoat (membrane) of the virus fuses with the cell membrane. Genetc code - written in RNA - from the virus passes into the cell, instructing a cellular machine called a ribosome to begin 


\section{Open Access Journal of Pulmonary \& Respiratory Sciences}

creating more copies of the virus genetic code.

Soon the cell's machinery is hijacked by the demands of the viral RNA to make virus membrane and spike proteins. When these wrap around the RNA, a new virus is created.

These collect in bubbles, called vesicles that move to the surface of the cell and spill out. New virus then spread into your lungs, throat, and mouth.

\section{What are the Symptoms of COVID-19?}

Based on 55,924 laboratory confirmed cases, typical signs and symptoms include: fever (87.9\%), dry cough (67.7\%), fatigue (38.1\%), sputum production (33.4\%), shortness of breath (18.6\%), sore throat (13.9\%), headache (13.6\%), joint or muscle pain (14.8\%), chills (11.4\%), nausea or vomiting $(5.0 \%)$, nasal congestion $(4.8 \%)$, and diarrhea $(3.7 \%)$.

\section{Do all Infected People Show Symptoms?}

No. Scientists are rushing to estimate how many people with mild or no symptoms that could be spreading the disease and by one estimate asymptomatic or mild cases combined represent up to half of all infections.

\section{How long does it take to Realize you have an Infection?}

The incubation period between infecton and symptoms appearing can range from two to 14 days. According to one study, about five days is the median (middle value) incubation period, while others put the average at around five days.

\section{Was it a Human-Made Virus?}

No. Analysis of the genetic sequences of the coronavirus responsible for the outbreak has confirmed the virus is natural and was not made in a lab.

\section{How did this Virus Cause a Pandemic?}

Coronaviruses are one of the families of virus that cause the common cold but, when it comes to SARS-CoV-2, we lack immunity because this particular virus came from another species. Viruses that circulate in animals keep jumping over to infect humans as we continue to plunder ecosystems: that was the story of HIV, of SARS, of MERS, of Ebola and now COVID-19. That is why scientists have warned that a pandemic of this magnitude was inevitable.

This virus is closely related to bat and pangolin corona viruses, perhaps a blend of bat and pangolin viruses that emerged by a process, called recombination, in a bat, pangolin or another species. (Note that MERS and SARS are also corona viruses with epidemic potential). The roots of the pandemic go back to when a cluster of pneumonia cases of unknown cause was reported by health authorities in Wuhan, China, in December 2019.

Further work revealed a link to the Huanan Seafood Wholesale Market, which also sold live animals. However, the Wuhan authorities were slow to report cases which delayed measures to contain it, and likely to have helped launch the pandemic.

\section{When can an Infected Person Infect Others?}

What is worrisome is that virus shedding, as detected in the mouth or nose, is common and could occur prior to onset of symptoms and can continue for days up to weeks after a person recovers.

\section{Is it very Contagious?}

Each case of measles causes 12 to 18 new cases, compared to about six for polio, smallpox and rubella, 1.4 for the 1918 influenza and around three for SARS-CoV-2, though this number (called the reproduction number) varies from person to person.

\section{How does the Virus Spread?}

Coronaviruses are generally transmitted by droplets through sneezing, coughing and when someone talks. Those droplets can then be inhaled by a person in close proximty or land on surfaces that others come into contact with, who can then get infected when they touch their eyes, nose, or mouth. However, evidence is beginning to emerge that the droplets may be so small that they can lead to airborne transmission over greater distances than originally thought.

\section{Is the Virus also Spread by Food?}

There is no evidence that food is a route of transmission.

\section{How deadly is Coronavirus?}

The overall fatality risk of COVID-19 in Wuhan, China, after developing symptoms was $1.4 \%$ as of 29 February 2020, according to a paper published in Nature Medicine. This figure is substantially lower than previously thought.

The death rate also depends on whether (as in the UK) you divide the number of deaths by the number of people 


\section{Open Access Journal of Pulmonary \& Respiratory Sciences}

admitted to hospital, or also include the much larger numbers of people with mild and moderate disease, revealed by extensive testing.

\section{What are Covid-19 Patients actually dying of?}

Most people will recover within a few days. But some go on to develop pneumonia and may need a ventilator to help them breathe. $\mathrm{n}$ severe cases, there is respiratory failure, septic shock, and/or multi-organ failure. Patients can suffer a fatal cytokine storm in which the immune system goes into overdrive, overwhelming the body with immune cells and the biochemical they make.

\section{Who is most at Risk?}

The risk of severe illness and death from COVID-19 is highest in older people, but the US Centers for Disease Control and Prevention says that millennials are by no means invincible: up to one-fifth of infected people aged 20-44 have been ill enough to be hospitalized; $2-4 \%$ of these required treatment in an intensive-care unit.

\section{Does this Virus Mutate Much?}

Yes. SARS-CoV-2 Asia so-called RNA virus, which means that its genetic material is RNA rather than the more familiar DNA found in everything from bacteria to humans. A characteristic of RNA viruses is their high rate of genetic mutation, which is a mechanism by which viruses evolve into new strains to escape host defenses.

Like the DNA in our cells, RNA consists of strings of chemicals - you can think of them as 'letters' -that spell out the genetic code, the instructions to build and run an organism. Prof Chris Whitty says that, as a rule, viruses tend to become less virulent 'as they become adapted to a new host'.

Some have referred to the 'deadly second wave' of the 1918 influenza pandemic, as a warning that worse is to come. That is apocryphal and, as this analysis concludes: 'remember that, as Kierkegaard said about life, influenza epidemics are lived forwards and understood backwards'.

\section{Cleaning up Coronavirus: How long does the Virus Hang about?}

The stability of this virus is cause for concern-it remains for several hours to days on surfaces and in droplets, suggesting that people may acquire the coronavirus through the air and after touching contaminated objects.
The SARS-CoV-2 virus is detectable in aerosols for up to three hours, up to four hours on copper, up to 24 hours on cardboard and up to two to three days on plastic and stainless steel, according to a recent study (summary).

\section{Is Coronavirus Transmitted by Drinking Water, Pools and Hot tubs?}

The virus has not been detected in drinking water and there is no evidence it can be spread through swimming pools that are properly operated, maintained and disinfected.

\section{Do Normal Cleaning Methods work with Coronavirus?}

Yes. This list highlights the disinfectants that meet the US Environmental Protection Agency criteria.

\section{Can i Make my own Alcohol Hand Sanitiser?}

Yes. My colleagues use this recipe from the World Health Organization to make bootleg sanitizer.

\section{How to end the Pandemic: How can we Treat and Prevent COVID-19?}

The global scientific community has mobilized to an extraordinary and unprecedented extent. Tens of thousands of scientists are redeploying to fight coronavirus. By 12 March, \#COVID19 had prompted the publication of more than 900 English-language scientific papers, preprints and reports.

They are using three basic approaches to fight the virus:

1. Drugs are typically small molecules that block various enzymes of the coronavirus to disable it. As one example, a potential coronavirus drug remdesivir blocks a particular enzyme that is required for the virus to replicate because it looks like a letter of RNA code to the enzyme but does not behave like it. Or a drug can work by blocking a protein that the virus uses to invade human cells.

2. Antibodies that neutralize the virus are found in survivors of the disease and can be used to jump-start an immune response in new patients, or engineered in the laboratory. For example, the company Takeda is developing hyper immune globulins, infection-fighting proteins isolated from the blood plasma of recovered patients, and Regeneron has isolated hundreds of virusneutralizing, fully human antibodies from mice which have been genetically-modified to have a human immune system. 


\section{Open Access Journal of Pulmonary \& Respiratory Sciences}

3. Vaccines, which have saved many millions of lives, introduce the inactivated virus or parts of it to the immune system to train the body's defenses to recognize them as an invader so, if ever exposed, the body knows how to fight the infection. Note that vaccines provide the body with a "memory" of how to fight the virus, unlike drugs and antibodies.

The first human clinical trial for a potential coronavirus vaccine, developed by the drug company Moderna, has started. Inovio Pharmaceuticals plans safety trials for its SARS-CoV-2 vaccine in April, as does a team at the University of Oxford, led by Sarah Gilbert.

However, even when a promising candidate is found, safety testing and mass manufacture takes time and money in the case of a new drug, it can take a decade or longer and cost around $\$ 2.6$ billion. In the case of vaccines, one radical proposal to speed their deployment is that scientists infect healthy people with the coronavirus and study whether those who get a prototype vaccine escape infection.

\section{Could an Existing drug be used to Treat Coronavirus?}

Quite possibly. The hunt is on for old drugs that might work against coronavirus, for instance by the World Health Organization and a consortium of 22 laboratories at the University of California, San Francisco.

A decades-old malaria drug, hydroxychloroquine and a closely-related medicine called chloroqune are of interest, though scientists do not share the optimism of President Trump that they are "one of the biggest game changers in the history of medicine," based on the current limited clinical data. There is also interest in repurposing drugs such as remdesivir, Favilavir and ribavirin, developed to combat RNA viruses, which include coronavirus.

In China, Favilavir has been approved by the National Medical Products Administration for use against the disease and 30 existing medicines are being tested to see if they may work.

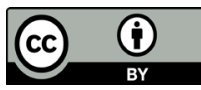

